\title{
Perioperative Management of Factor V Leiden and Pancreatic Adenocarcinoma
}

\author{
Thomas J. O'Malley, ${ }^{1,2}$ Renganaden Sooppan, ${ }^{1,2}$ and Charles J. Yeo, ${ }^{1,2, *}$
}

\begin{abstract}
Background: Perioperative management of a patient with factor $\vee$ Leiden has been infrequently reported with most studies focused in the orthopedic literature.

Case Presentation: We report a case of a 74-year-old woman who underwent a pylorus-preserving pancreaticoduodenectomy without immediate perioperative embolic or thrombotic phenomena and her management throughout her hospital course.

Conclusion: To the best of our knowledge, this is the first known report regarding factor $V$ Leiden and surgical management of pancreatic ductal adenocarcinoma.
\end{abstract}

Keywords: factor V Leiden; hypercoagulability; pancreaticoduodenectomy

\section{Introduction}

Factor V Leiden, the most common heritable hypercoagulability disorder, represents a unique perioperative challenge for surgeons today. Much of the current literature focuses on the medical management of this condition, yet few articles discuss appropriate management of factor $\mathrm{V}$ Leiden in major abdominal surgery. We present a case of a patient with factor V Leiden who underwent a successful pancreaticoduodenectomy.

\section{Case Report}

A 74-year-old woman presented to her primary care provider with complaints of pruritus, tea-colored urine, pale stools, and jaundice for 1 week. She additionally reported early satiety, decreased oral intake, and an unintentional seven-pound weight loss. At an outside facility, her hepatic function panel showed a total bilirubin of $13.0 \mathrm{mg} / \mathrm{dL}$, a direct bilirubin of $11.8 \mathrm{mg} / \mathrm{dL}$, an AST of $281 \mathrm{U} / \mathrm{L}$, an ALT of $401 \mathrm{U} / \mathrm{L}$, and a serum CA 19-9 returned significantly elevated at $900 \mathrm{U} / \mathrm{mL}$ (normal: 0-37 U/mL). Her abdominal CT scan showed a mass suspicious for pancreatic adenocarcinoma (Fig. 1). She was promptly transferred to our facility. Her medical history is notable for factor V Leiden hypercoagulability disorder with associated deep vein thrombosis in 2003 and 2010, both of which resulted in pulmonary embolism, a history of breast cancer, hypertension, hyperlipidemia, carotid stenosis, and gastroesophageal reflux disease. Her surgical history includes a right breast lumpectomy and sentinel lymph node biopsy with radiation therapy and a laparoscopic hysterectomy. Her family history is significant for factor V Leiden hypercoagulability disorder in her brother as well as her sister, both of whom have developed deep vein thrombosis and subsequent pulmonary embolism. Her physical examination before surgery revealed a soft, nontender, nondistended abdomen and was only significant for cutaneous jaundice and icteric sclera.

At our facility, she underwent an esophagogastroduodenoscopy and endoscopic ultrasonography with fine needle aspiration, which revealed a 2.8 by $2.0 \mathrm{~cm}$ uncinate process pancreatic mass staged sonographically as a T3N1Mx mass. The fine-needle aspiration retrieved material consistent with pancreatic adenocarcinoma. During this procedure, she underwent a biliary sphincterotomy and placement of a 10F plastic biliary endoprosthesis. In

\footnotetext{
${ }^{1}$ Department of Surgery, The Sidney Kimmel Medical College at Thomas Jefferson University, Philadelphia, Pennsylvania.

${ }^{2}$ Jefferson Pancreas, Biliary and Related Cancer Center, Philadelphia, Pennsylvania. 


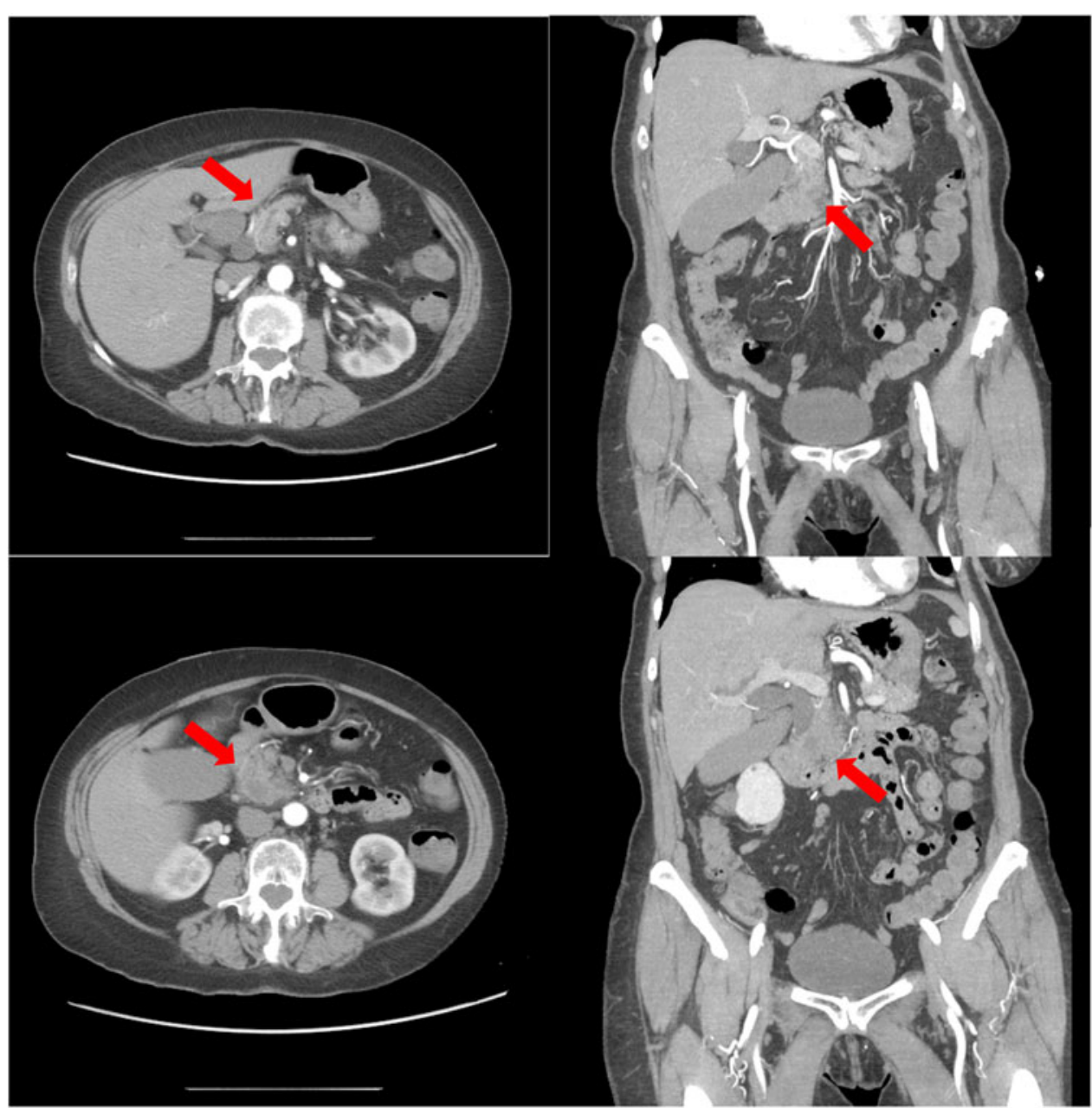

FIG. 1. Representative axial and coronal $C T$ images with red arrow showing a pancreatic head mass with biliary duct, pancreatic duct, and gall bladder dilation.

preparation for her operation, a specialized group at our facility known as the Jefferson Anti-Thrombotic Service (JATS) was consulted for assistance in management of her hypercoagulability disorder. The patient had previously been on warfarin with maintenance of a therapeutic international normalized ratio of 2-3; however, her levels had recently been erratic likely secondary to her biliary obstruction and jaundice. At this time, it was deemed appropriate for the patient to be placed on a therapeutic enoxaparin dose of $60 \mathrm{mg}$ every $12 \mathrm{~h}$ that would be discontinued $36 \mathrm{~h}$ before surgery. She was discharged home after bile duct stent placement with her operation scheduled for the following week.

Preoperatively, the patient was given a single dose of $5000 \mathrm{U}$ of heparin, T.E.D. antiembolism stockings were placed, and intermittent pneumatic compression de- vices were placed as is the standard venous thromboembolism propylaxis protocol at our facility. The patient then underwent an uncomplicated pylorus-preserving pancreaticoduodenectomy and open cholecystectomy. Postoperatively, the JATS team followed the patient and recommended starting a prophylactic dose of heparin $5000 \mathrm{U}$ every $8 \mathrm{~h}$ or enoxaparin $40 \mathrm{mg}$ each day $12-$ $24 \mathrm{~h}$ after the operation and to use adjunctive methods such as T.E.D. stockings and intermittent pneumatic compression. We elected to begin enoxaparin beginning on postoperative day 1 , and continued the adjunctive methods of stockings and compression devices, which had commenced in the operating room.

The patient recovered well from her operation and was continued on her antithrombotic regimen without evidence of venous or arterial embolic or thrombotic 
events throughout her hospital stay. Her final pathology revealed a $2.6 \mathrm{~cm}$ invasive, poorly differentiated pancreatic ductal adenocarcinoma with focal signet ring and clear cell features, with 12 specimen lymph nodes negative for malignancy, and negative surgical margins. She was discharged home with instructions to take a daily prophylactic dose of $40 \mathrm{mg}$ enoxaparin until her follow-up appointment, which she did. Approximately 3 weeks after her discharge, she developed chest pain, presented to an outside hospital, and was found to have a pulmonary embolism. She was monitored for several days at the outside hospital and in discussion with her providers underwent treatment with a therapeutic dosing of enoxaparin $60 \mathrm{mg}$ twice a day. She had an uneventful recovery from this episode and was transitioned from enoxaparin to rivaroxaban at her follow-up with JATS in March 2017.

\section{Discussion}

Although this scenario represents one of the first known reports of a patient with factor V Leiden hypercoagulability disorder undergoing pancreatic resection for adenocarcinoma, these respective conditions are not uncommon. Factor V Leiden represents the most common inherited hypercoagulability disorder with 1 in 5000 people having the condition. ${ }^{1}$ Pancreatic cancer is currently the third leading cause of cancer death in the United States and the intersection of these two diseases will most certainly be encountered in the future by clinicians. It is, therefore, important to understand the pathogenesis of factor V Leiden and its relation to perioperative complications.

In the normal population, activated protein $\mathrm{C}$, an anticoagulant, binds to factors Va and VIIIa and cleaves them resulting in their inactivation. This interaction prevents further generation of thrombin, thus resulting in an anticoagulation mechanism. ${ }^{2}$ Factor V Leiden results from a genetic inheritance of a nucleotide substitution that alters the cleavage site of activated protein $\mathrm{C}$. Without this locus, factor Va cannot be inactivated by activated protein $\mathrm{C}$ and this mechanism of anticoagulation is lost, thus resulting in excessive thrombin formation (Fig. 2).

Clinical manifestations of factor $\mathrm{V}$ Leiden include recurrent venous thromboembolism, complications of pregnancy, pulmonary embolism, and a smaller association with arterial thromboembolism. ${ }^{3-6}$ Our patient suffered two episodes of deep vein thrombosis and two episodes of pulmonary embolism before her operation as well as one episode postoperatively. These complications require treatment often involving anticoagulation medications. The first event is usually managed by low molecular weight heparin or a heparin-to-warfarin bridge for 3 months at a minimum. ${ }^{7}$ Anticoagulation for recurrent events in factor $\mathrm{V}$ Leiden populations may increase bleeding risk as the complications of thromboembolism are treated. This presents a perioperative challenge for the surgeon who must balance between hemostasis and the risk of thrombosis.

Several articles have examined the effect of factor V Leiden and its consequences on hypercoagulable events postoperatively. Despite extensive review and the theoretical risk of hypercoagulable disorders, these studies indicate that patients with factor $\mathrm{V}$ Leiden demonstrate no increased risk for a perioperative complication related to hypercoagulability, yet their long-term risk as it relates to clotting remains higher than the normal population. ${ }^{5,8,9}$ In a gynecological oncology population, factor $\mathrm{V}$ Leiden was deemed to not carry increased thrombosis risk despite solid tissue malignancies. ${ }^{10}$ However, because anticoagulation medications and mechanical interventions are often part of the standard of care after an operation, the increased risk a patient might carry because of a hypercoagulable disorder may be outweighed by the standard perioperative interventions. Although it may be necessary to pay particular attention to patients with factor $\mathrm{V}$ Leiden in the perioperative population, routinely testing patients to assess whether or not they possess the factor V Leiden locus is not recommended as testing for thrombophilia does not reduce the risk of recurrent venous thromboembolism, although not all reports agree. ${ }^{1,11-14}$ Thus although factor $\mathrm{V}$ Leiden may contribute to venous thrombosis, most current literature indicates that the increased risk of hypercoagulability conferred by factor V Leiden is outweighed by current surgical management, including the use of chemo- and mechanoprophylaxis of venous thrombosis in the postoperative period. ${ }^{15}$ However, most agree that patients who experience multiple thrombotic events must be placed on a long-term anticoagulation regimen after the perioperative period, as was done with this patient. ${ }^{16,17}$ Hereditary thrombophilia was not considered to be significant enough to alter the time course of antithrombotic therapy, and heterozygosity of factor $\mathrm{V}$ Leiden by itself is not an indication for long-term anticoagulation without associated indications. ${ }^{1}$ According to the recommended guidelines recently published in CHEST, patients who suffer from an unprovoked pulmonary embolism (PE) or deep venous thrombosis (DVT) for the first time should be treated with vitamin $\mathrm{K}$ antagonists for 3 months and are then evaluated for the risks to benefits of indefinite therapy. Furthermore, they recommend indefinite therapy for patients with a 


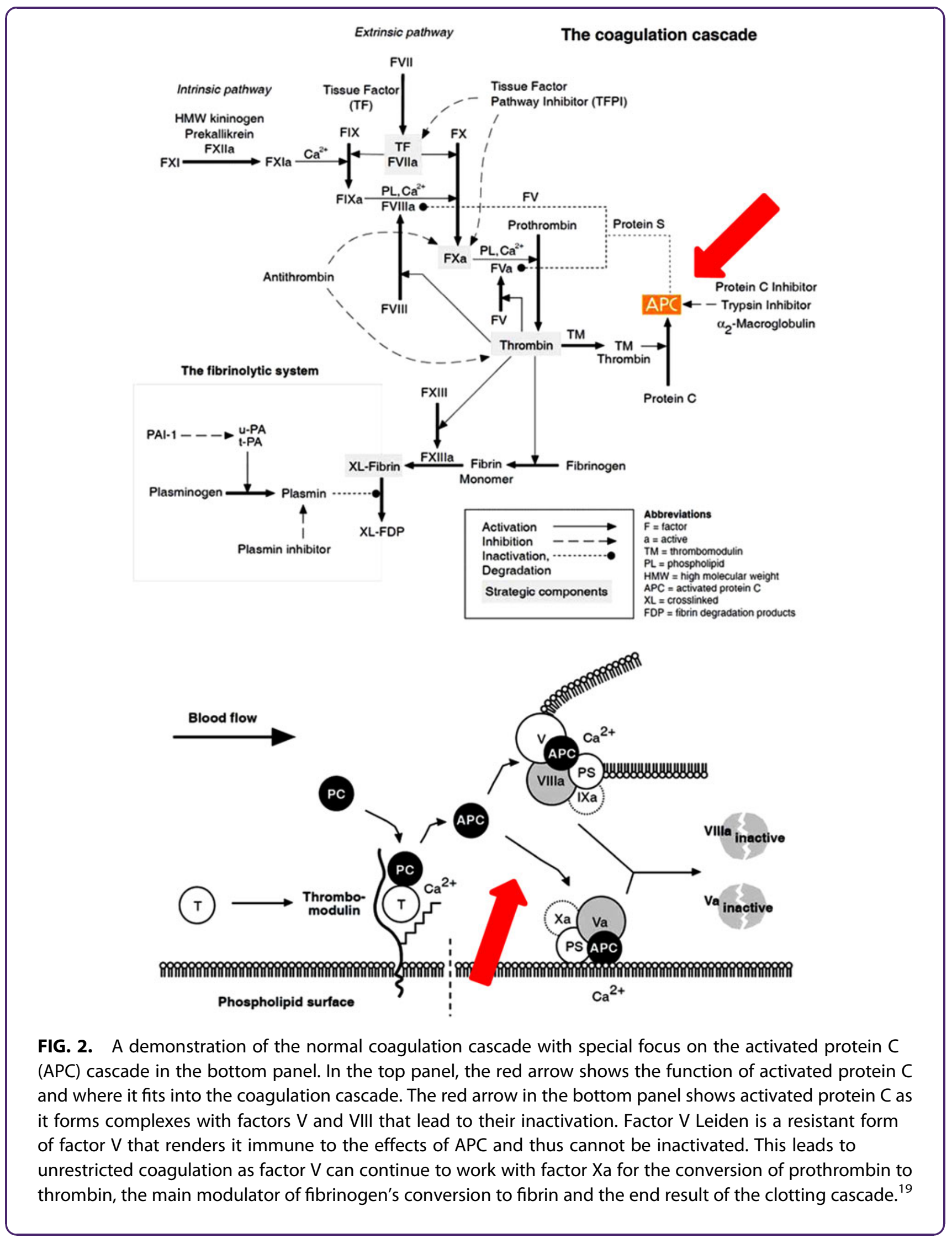


first unprovoked proximal DVT or PE and a low risk of bleeding, and for patients with a second unprovoked DVT. ${ }^{18}$ As this patient had suffered multiple thrombotic episodes, her treatment with indefinite anticoagulation medications was deemed necessary.

The development of a pulmonary embolism in this patient was an unfortunate complication of the treatment of a complex disease. Although the patient suffered no early postoperative thrombotic complications, the embolism managed to occur despite prophylactic enoxaparin dosing. This seems to suggest that part of her management should have including transitioning to a therapeutic enoxaparin dose. The exact moment in which this transition should be made is difficult to assess. In this particular case, the decision of which dose to place the patient on was driven largely by the expert advice of our consultants with JATS. Our limited experience with disease process suggests that factor $\mathrm{V}$ Leiden patients should return to their therapeutic anticoagulant medications as soon as they are safely able to in the postoperative period, and in this particular case, we would suggest placing patients on their therapeutic anticoagulation within 3 weeks of their operation.

Based on a current review of the literature and our experience with the management of this patient, we would recommend routine chemoprophylaxis in the immediate postoperative period with enoxaparin or heparin as well as mechanoprophylaxis including T.E.D. antiembolism stockings and intermittent pneumatic compression devices to prevent thrombosis in the factor $V$ Leiden population and the perioperative patient population at large. After the immediate postoperative period for patients at increased risk for venous thromboembolism, we recommend the patient take a daily anticoagulation medication and be followed by a hypercoagulable disorder specialist.

\section{Author Disclosure Statement}

No competing financial interests exist.

\section{References}

1. Kujovich JL. Factor V Leiden Thrombophilia. Genet Med. 2011;13:1-16.

2. Martlew VJ. Peri-operative management of patients with coagulation disorders. Br J Anaesth. 2000;85:446-455.

3. Bloomenthal D, Delisle MF, Tessier F, et al. Obstetric implications of the factor V Leiden mutation: a review. Am J Perinatol. 2002;19:37-47.

4. Simioni $P$, Prandoni $P$, Lensing AW, et al. Risk for subsequent venous thromboembolic complications in carriers of the prothrombin or the factor $\mathrm{V}$ gene mutation with a first episode of deep-vein thrombosis. Blood. 2000;96:3329-3333.

5. Blaszyk H, Bjornsson J. Factor $\mathrm{V}$ Leiden and morbid obesity in fatal postoperative pulmonary embolism. Arch Surg. 2000;135:1410-1413.

6. Szolnoki Z, Somogyvari F, Kondacs A, et al. Evaluation of the roles of the Leiden $\mathrm{V}$ mutation and ACE I/D polymorphism in subtypes of ischaemic stroke. J Neurol. 2001;248:756-761.
7. Galioto NJ, Danley DL, Van Maanen RJ. Recurrent venous thromboembolism Am Fam Physician. 2011;83:293-300.

8. Ryan DH, Crowther MA, Ginsberg JS, et al. Relation of factor V Leiden genotype to risk for acute deep venous thrombosis after joint replacement surgery. Ann Intern Med. 1998;128:270-276.

9. Wahlander K, Larson G, Lindahl TL, et al. Factor V Leiden (G1691A) and prothrombin gene G20210A mutations as potential risk factors for venous thromboembolism after total hip or total knee replacement surgery. Thromb Haemost. 2002;87:580-585.

10. Ravin AJ, Edwards RPA, Krohn M, et al. The factor V Leiden mutation and the risk of venous thromboembolism in gynecologic oncology patients. Obstet Gynecol. 2002;100:1285-1289.

11. Lowe GD, Haverkate F, Thompson SG, et al. Prediction of deep vein thrombosis after elective hip replacement surgery by pre-operative clinical and haemostatic variables: the ECAT DVT Study-European Concerted Action on Thrombosis. Thromb Haemost. 1999;81:879-886.

12. Eckman MH, Erban JK, Singh SK, et al. Screening for the risk for bleeding or thrombosis. Ann Intern Med. 2003;138:15-24.

13. Atluri $P$, Raper SE. Factor $V$ Leiden and postoperative deep vein thrombosis in patients undergoing open Roux-en-Y gastric bypass surgery. Obes Surg. 2005;15:561-564.

14. Donahue BS. Factor V Leiden and perioperative risk. Anesth Analg. 2004;98:1623-1634.

15. Massoudy $P$, Thielmann $M$, Muller-Beissenhirtz $H$, et al. Thrombophilia in cardiac surgery-patients with symptomatic factor V Leiden. J Card Surg. 2009;24:379-382.

16. Pabinger I, Ay C, Dunkler D, et al. Factor V Leiden mutation increases the risk for venous thromboembolism in cancer patients-results from the Vienna Cancer and Thrombosis Study (CATS). J Thromb Haemost. 2015;13:17-22.

17. Coppens $M$, Reijnders $\mathrm{JH}$, Middeldorp S, et al. Testing for inherited thrombophilia does not reduce the recurrence of venous thrombosis. J Thromb Haemost. 2008;6:1474-1477.

18. Kearon C, Kahn SR, Agnelli G, et al. Antithrombotic therapy for venous thromboembolic disease: American College of Chest Physicians EvidenceBased Clinical Practice Guidelines (8th Edition). Chest. 2008;133:454S-545S

19. Activated Protein C (APC) and Factor V Leiden. (2017, April 2). Available at http://diapharma.com/activated-protein-c-apc-factor-v-leiden (accessed June 1, 2017).

Cite this article as: O'Malley TJ, Sooppan R, Yeo CJ (2017) Perioperative management of factor $\mathrm{V}$ Leiden and pancreatic adenocarcinoma, Journal of Pancreatic Cancer 3:1, 53-57, DOI: 10.1089/ pancan.2017.0010.

\section{Abbreviation Used \\ JATS $=$ Jefferson Anti-Thrombotic Service}



